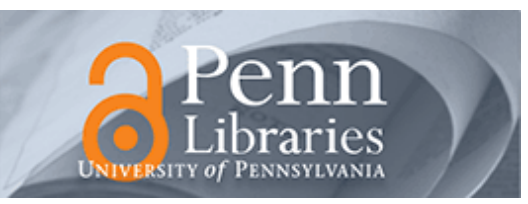

University of Pennsylvania ScholarlyCommons

February 2003

\title{
Magnetically aligned single wall carbon nanotube films: preferred orientation and anisotropic transport properties
}

John E. Fischer

University of Pennsylvania, fischer@seas.upenn.edu

Wei Zhou

University of Pennsylvania

Juraj Vavro

University of Pennsylvania

Mark C. Llaguno

University of Pennsylvania

Csaba Guthy

University of Pennsylvania

See next page for additional authors

Follow this and additional works at: https://repository.upenn.edu/mse_papers

\section{Recommended Citation}

Fischer, J. E., Zhou, W., Vavro, J., Llaguno, M. C., Guthy, C., Haggenmueller, R., Casavant, M. J., Walters, D. E., \& Smalley, R. E. (2003). Magnetically aligned single wall carbon nanotube films: preferred orientation and anisotropic transport properties. Retrieved from https://repository.upenn.edu/mse_papers/62

Postprint version. Published in Journal of Applied Physics, Volume 93, Issue 4, 15 February 2003, pages 2157-2163.

Publisher URL: http://dx.doi.org/10.1063/1.1536733

This paper is posted at ScholarlyCommons. https://repository.upenn.edu/mse_papers/62

For more information, please contact repository@pobox.upenn.edu. 


\title{
Magnetically aligned single wall carbon nanotube films: preferred orientation and anisotropic transport properties
}

\author{
Abstract \\ Thick films of single wall carbon nanotubes (SWNT) exhibiting in-plane preferred orientation have been \\ produced by filter deposition from suspension in strong magnetic fields. We characterize the field-induced \\ alignment with x-ray fiber diagrams and polarized Raman scattering, using a model which includes a \\ completely unaligned fraction. We correlate the texture parameters with resistivity and thermal \\ conductivity measured parallel and perpendicular to the alignment direction. Results obtained with 7 and \\ 26 Tesla fields are compared. We find no significant field dependence of the distribution width, while the \\ aligned fraction is slightly greater at the higher field. Anisotropy in both transport properties is modest, \\ with ratios in the range $5-9$, consistent with the measured texture parameters assuming a simple model \\ of rigid rod conductors. We suggest that further enhancements in anisotropic properties will require \\ optimizing the filter deposition process rather than larger magnetic fields. We show that both $\mathrm{x}$-ray and \\ Raman data are required for a complete texture analysis of oriented SWNT materials.
}

\section{Comments}

Postprint version. Published in Journal of Applied Physics, Volume 93, Issue 4, 15 February 2003, pages 2157-2163.

Publisher URL: http://dx.doi.org/10.1063/1.1536733

\section{Author(s)}

John E. Fischer, Wei Zhou, Juraj Vavro, Mark C. Llaguno, Csaba Guthy, Reto Haggenmueller, M. J. Casavant, D. E. Walters, and Richard E. Smalley 


\title{
Magnetically aligned single wall carbon nanotube films: preferred orientation and anisotropic transport properties
}

\author{
J. E. Fischer ${ }^{\text {a) }}$, W. Zhou, J. Vavro, M. C. Llaguno, C. Guthy, R. Haggenmueller \\ Department of Materials Science and Engineering and \\ Laboratory for Research on the Structure of Matter \\ University of Pennsylvania, Philadelphia PA 19104-6272 \\ M. J. Casavant, D. E. Walters b) and R. E. Smalley \\ Center for Nanoscale Science and Technology \\ Rice University, Houston TX 77259
}
a) fischer@1rsm.upenn.edu
b) Current address: Department of Physics, University of Central Florida, Orlando FL 32816-2385
PACS numbers : 78.70.Nx, 61.46.+w, 61.48.+c




\begin{abstract}
Thick films of single wall carbon nanotubes (SWNT) exhibiting in-plane preferred orientation have been produced by filter deposition from suspension in strong magnetic fields. We characterize the field-induced alignment with x-ray fiber diagrams and polarized Raman scattering, using a model which includes a completely unaligned fraction. We correlate the texture parameters with resistivity and thermal conductivity measured parallel and perpendicular to the alignment direction. Results obtained with 7 and 26 Tesla fields are compared. We find no significant field dependence of the distribution width, while the aligned fraction is slightly greater at the higher field. Anisotropy in both transport properties is modest, with ratios in the range 5-9, consistent with the measured texture parameters assuming a simple model of rigid rod conductors. We suggest that further enhancements in anisotropic properties will require optimizing the filter deposition process rather than larger magnetic fields. We show that both x-ray and Raman data are required for a complete texture analysis of oriented SWNT materials.
\end{abstract}

\title{
INTRODUCTION
}


Single wall carbon nanotube (SWNT) materials can be partially aligned by mechanical shear [1], anisotropic flow [2], gel extrusion [3,4] or filter deposition from suspension in strong magnetic fields [5]. One of the motivations is to obtain the highest possible fraction of the excellent intrinsic axial properties of SWNT in a real material. We reported previously on the texture and properties of SWNT films deposited at 26 Tesla using a resistive solenoid located at the National High Magnetic Field Laboratory in Florida [6,7]. This approach is not practical for large-scale production. It was later found that aligned films could be achieved in the laboratory using a 7 Tesla superconducting solenoid [8]. Here we compare the 7 Tesla texture and properties with those obtained at 26 Tesla. We use both x-ray fiber diagrams and orientation-dependent polarized Raman scattering [2,9] to characterize the magnetic field-induced alignment. The data are analyzed with a "2-phase" model consisting of an aligned fraction characterized by the angular distribution of tube axes about the preferred direction, plus a completely unaligned fraction to account for SWNT aggregates which are insufficiently dispersed to respond to the aligning field [2]. This approach was previously applied to texture studies of oriented films and fibers of conjugated polymers [10,11]. The deduced SWNT texture is correlated with measurements of electrical resistivity $\rho$ and thermal conductivity $\kappa$ parallel and perpendicular to the preferred direction. Differences between 7 and 26 Tesla alignment give some clues about the alignment mechanism and strategies for improving the process.

Magnetic alignment in suspension requires that the energy produced by the torque acting on a magnetically anisotropic segment exceed the thermal energy: $\delta U \sim \mathrm{B}^{2} \mathrm{n} \delta \chi>$ $\mathrm{kT}$ where $\mathrm{B}$ is the field strength, $\mathrm{n}$ is the number of carbon atoms in the segment and $\delta \chi$ 
is the magnetic anisotropy [5]. For fixed $\mathrm{B}$ and $\mathrm{n}, \delta \mathrm{U}$ is comparable for metallic and semiconducting SWNT. Specifically, for a single $300 \mathrm{~nm}$ long $(10,10)$ tube segment $\delta U$ $=5 \mathrm{kT}$ at 15 Tesla. The actual suspension is polydisperse, and $\mathrm{n}$ will be small for short single tubes and large for long bundles. Thus the latter should align at smaller fields; similarly 26 Tesla should be $\sim 14$ times more effective than 7 Tesla for fixed $n$.

Samples were prepared as described previously $[5,8]$. The starting material [12]

was grown by pulsed laser ablation at $1100^{\circ} \mathrm{C}$, purified and filter-deposited from suspension in the magnetic field. Robust films with strikingly obvious preferred cleavage directions were removed from the filter membranes, then annealed in vacuum at $1150^{\circ} \mathrm{C}$ using a slow ramp to drive off volatiles and enhance crystallinity.

\section{X-RAY SCATTERING}

The X-ray scattering intensity from a bulk SWNT sample generally contains 3 contributions: a crystalline component (Bragg peaks from ordered bundles, or "ropes", plus any crystalline impurities); a non-crystalline component (diffuse scattering from isolated tubes, small bundles and poorly crystallized large bundles), and small-angle scattering (SAXS) from uncorrelated pores, impurity particles etc. Due to the onedimensional character of isolated tubes and the two-dimensional rope lattice [13], both Bragg and diffuse scattering contain information related to preferred orientation. The rope peaks are broadened by diameter dispersion and by the finite and variable number of tubes per rope [14]. The diffuse contribution from SWNT is complicated by the influence of defects, nanotube curvature and finite tube length as well as diameter 
dispersion. SWNT-related diffuse scattering cannot be separated from small-angle scattering (SAXS) contrast originating in porosity or nanoscopic particles of graphite, metal catalysts, amorphous carbon, graphitic onions etc. Therefore it has proved impossible to determine fractional crystallinity from the relative intensities of Bragg and diffuse scattering.

Texture determination from $\mathrm{x}$-ray fiber diagrams has two major advantages over polarized Raman scattering. It is unquestionably a bulk measurement for thin foils in transmission, and it unambiguously reveals the width of the distribution in the plane of the film sample. Diffraction from crystalline ropes shares the major advantage of Raman scattering, namely a response which is specific to nanotubes. Low-angle diffuse scattering can also give useful information about texture as long as SAXS from the extraneous diffuse scatterers is isotropic.

The importance of diffuse scattering in SWNT materials is illustrated by a survey experiment carried out on a horizontal one-dimensional diffractometer equipped with a linear detector covering $120^{\circ}$ in $2 \theta[12,13]$. The sample was mounted in reflection geometry at grazing incidence (fixed sample angle), with the preferred axis vertical (normal to the diffracting plane) giving maximum sensitivity to 2-D rope reflections. A typical profile is shown in Figure 1. The 4 broad peaks are associated with Bragg reflections from the rope lattice $[12,13]$ while the sharp peak at $26.4^{\circ}$ represents a small amount of residual graphite impurity. We determined that a substantial fraction of the monotonic "background" is diffuse scattering from the sample. This sample is not very 
crystalline; the intensity ratio of the first rope peak to "background" is $\sim 1: 3$ while ratios as high as 5:1 have been observed. We estimate that half the intensity at $6.3^{\circ}$ consists of air scattering while about $1 / 4$ each can be attributed to sample-related diffuse scattering (not necessarily all from SWNT) and Bragg scattering from semi-crystalline ropes.

Texture analysis was performed using the shortest sample-detector distance available on a multi-angle diffractometer [15] equipped with $\mathrm{Cu}$ rotating anode, doublefocussing optics, evacuated flight path ( $\sim 8 \mathrm{mbar})$ and 2-D wire detector. Single films 4-7 $\mu \mathrm{m}$ thick were measured in transmission for 1 or 2 hours. The $\mathrm{x}$-ray spot size at the sample was $\sim 200 \mu$ in diameter. Background was recorded with no sample and simply subtracted off since absorption by the sample was negligible. Radial (20) cuts normal to the alignment axis (azimuth $\chi=0^{\circ}$ and $180^{\circ}$ ) resembled Figure 1, confirming the superposition of 2-D rope peaks and diffuse scattering. Preferred orientation was obtained by summing pixels in $2.4^{\circ}$ radial sectors $1^{\circ}$ wide in $\chi$, centered at $2 \theta=6.3^{\circ}$. From Figure 1 it is clear that these sectors include Bragg and diffuse scattering, so the $\chi$ dependence will include both crystalline and non-crystalline SWNT components in some unknown ratio. The results are plotted in Figure 2 for 7 Tesla (top) and 26 Tesla (bottom) samples. The solid curves are least square fits to Gaussians centered at $\chi=0$ and $180^{\circ}$ plus a constant, where the alignment direction runs through the $90^{\circ}-270^{\circ}$ axis. We define $A_{X}$ as the integrated intensity ratio of the Gaussian alone to Gaussian + constant, realizing that the constant includes contributions from unaligned tubes and isotropic non- 
SWNT constituents and therefore $A_{X}$ is not an accurate measure of the aligned fraction. The fitted FWHM's (full width at half-maximum) are $33.0 \pm 0.2^{\circ}$ and $34.0 \pm 0.5^{\circ}$ for 7 and 26 Tesla respectively; the corresponding $\mathrm{A}_{X}$ values are 0.42 and 0.40 . These are not significantly different for the two magnetic field strengths. Radial scans centered about $\chi=90^{\circ}$ and $270^{\circ}$ showed no Bragg intensity, therefore the entire crystalline component is aligned for both field strengths. Lorentzian fits were also performed and showed the same trends; the Gaussian distribution function is preferred for comparison with Raman data as described below.

We estimate the degree to which the above represents alignment of the crystalline component by shifting the $2 \theta$ window well below the Bragg peak to isolate texture in the diffuse scattering. Figure 3 shows the result for a 7 Tesla sample, with data summed over a $1.5^{\circ}$ radial sector centered at $3.7^{\circ}$. The Gaussian FWHM is $\sim 10^{\circ}$ broader than before, $42 \pm 1^{\mathrm{o}}$ compared to $33 \pm 0.2^{\circ}$, implying that single tubes and small ropes are not as well aligned as the larger crystalline ropes. This in turn means that $33^{\circ}$ is an upper bound for the mosaic spread of the crystalline component since the sectors centered at $6.3^{\mathrm{o}}$ include a substantial contribution from the less well-aligned non-crystalline tubes. The corresponding value of $\mathrm{A}_{X}$ is reduced to 0.36 (diffuse only) from 0.42 (Bragg plus diffuse). As noted above, $\mathrm{A}_{\mathrm{X}}$ (Bragg) is $\sim 1$ so the rule of mixtures would say that most of the aligned SWNT material is noncrystalline. The fallacy in this reasoning is that the 
diffuse scattering at all $\chi$ is enhanced by isotropic SAXS from pores and nanoparticles; thus the motivation for a SWNT-specific probe.

\section{RAMAN SCATTERING}

Using VV polarization and taking measurements at many angles $\Psi$ between the preferred axis (here the $\mathrm{H}$-vector) and polarization vector, one obtains a distribution function characteristic of tube axis orientations [9]. Raman scattering from SWNT does not rely on crystal structure and therefore detects all the tubes in the sample. It also strongly discriminates against non-SWNT constituents due to resonant enhancement [9]. Unlike the x-ray case, we cannot directly measure a unique component of the full 3-D "pole figure" because the Raman intensity depends on misalignment with respect to the plane defined by the preferred axis and polarization vectors, as well as within that plane. There are two limiting cases: axially-symmetric fibers where the two components are the same [16], and films in which the tubes are everywhere parallel to the film plane (out-ofplane FWHM =0). The materials studied here no doubt fall somewhere between these limits. We expect the out-of-plane mosaic to be narrower than the in-plane value (determined unambiguously by x-ray analysis) due to the additional driving force for alignment associated with the filter deposition geometry. For the general case of anisotropic 3-D texture, Raman scattering cannot be used to directly measure the two principal components. In our specific case of thin film geometry with strong X-ray scattering limited to small Bragg angles, the accuracy with which the out-of-plane FWHM can be measured is limited by angle-dependent corrections for scattering volume and sample absorption [17]. 
We analyze the Raman data using both a simple 2-D model in which out-of-plane misalignment is neglected, and an anisotropic 3-D model. We modified the original analysis [9] to include a completely unaligned fraction (1- $\left.A_{R}\right)$, and by using Gaussian rather than Lorentzian lineshapes to describe the preferred orientation. The power law decay of the Lorentzian leads to unacceptably strong correlation between $A_{R}$ and the distribution width in the least squares fits.

Raman peak intensities of the tangential Raman $\mathrm{G}_{2}$-band at $1590 \mathrm{~cm}^{-1}$ were averaged over 5 different $2 \mu \mathrm{m}$ spots to account for inhomogeneity, at each of 7 values of $\Psi$, the angle between the preferred axis in the sample plane and the polarization direction. Note that the sampled area is $\sim 10,000$ times smaller than the $\mathrm{x}$-ray value. We used $514.5 \mathrm{~nm}$ excitation and a Renishaw Ramanscope 1000 system. Background correction was achieved by subtracting a spectrum of amorphous carbon. In the 2-D model, the Raman intensity data were fitted to a function which describes the deviation from perfect alignment of $100 \%$ of the material:

$$
\mathrm{F}\left(\Psi, A_{R}, \sigma\right)=\frac{1-A_{R}}{\pi}+A_{R} \frac{1}{\sigma \sqrt{\pi / 2}} e^{-\frac{2 \Psi^{2}}{\sigma^{2}}}
$$

where $A_{R}=$ aligned fraction and $\sigma$ is the Gaussian standard deviation (equivalent $\mathrm{FWHM}=\sqrt{2 \ln 2} \sigma$ ). For $\sigma=0$ and $\mathrm{A}_{\mathrm{R}}=1, \mathrm{~F}$ reduces to zero and the Raman intensity is proportional to $\cos ^{4} \Psi$, vanishing at $\Psi=\pi / 2$. Two orthogonal measurements at $\Psi=0$ and $\pi / 2$ give a ratio which depends on both $\sigma$ and $A_{R}$. If $\sigma$ is small, $I(0) / I(\pi / 2)$ gives 
a zero-order estimate of $A_{R}$. In general, even with Raman data taken at many $\Psi$ 's, $\sigma$ is obtained by fitting the deviation from a $\cos ^{4} \Psi$ law. This requires very accurate intensities, and is an inherent limitation of the Raman method compared to x-ray fiber diagrams.

The 3-D model is a product of two independent 2-D distribution functions $\mathrm{F}_{\text {in-plane }}\left(\Psi, \mathrm{A}_{\mathrm{R}}, \sigma\right) \mathrm{F}_{\text {out-of-plane }}\left(\Psi^{*}, \mathrm{~A}_{\mathrm{R}}{ }^{*}, \sigma^{*}\right)$. As noted above, we expect $\sigma^{*}<\sigma$ and $\mathrm{A}_{\mathrm{R}}{ }^{*}>$ $A_{R}$ due to the tendency of tubes/ropes to conform to the flat substrate. We neglected the anisotropic optical penetration depth, mainly due to the lack of empirical data with which to treat it properly. The depth sampled by Raman scattering is greater in the weaklyabsorbing transverse orientation $\Psi=\pi / 2$ compared to $\Psi=0$. As a consequence $I(\pi / 2)$ is overestimated relative to $\mathrm{I}(0)$ etc., which means that $\sigma$ is overestimated and/or $A_{R}$ is underestimated.

In Figure 4 we show representative angle-dependent Raman spectra for the 7 Tesla sample. These generally exhibit 3 groups of peaks: the radial breathing modes just below $200 \mathrm{~cm}^{-1}$, the disorder-induced D-band centered at $1350 \mathrm{~cm}^{-1}$, and the tangential G-band modes which peak at $1590 \mathrm{~cm}^{-1}$. Note that the intensities of all 3 features exhibit the same orientation dependence [9]. Figure 5 shows the orientation dependence of the G-band peak intensity for 7 Tesla (a) and 26 Tesla (b) samples. Fits using the 2-D model with $\sigma$ and $A_{R}$ as independent parameters showed that $\sigma$ could be specified only to 
within $\sim 30 \%$ while the corresponding uncertainties in $A_{R}$ were only $5 \%$. This suggested using the $x$-ray in-plane FWHM to fix $\sigma$ and then to optimize $A_{R}$, thus combining the best features of both techniques. An example is shown in Figure 5 based on the anisotropic 3-D model, where we assume $\mathrm{A}_{\mathrm{R}}{ }^{*}=0.2$ and $\sigma^{*}=10^{\circ}$. Model curves (solid) with optimized in-plane aligned fractions reproduce the data (dots with error bars) quite well. The error bars are standard deviations of the 5-point averages. Note the large relative intensity at $\Psi=\pi / 2$, indicating significant fractions of unaligned material at both fields; the Raman ratios $\mathrm{I}(0) / \mathrm{I}(\pi / 2)$ are $\sim 3$ and $\sim 4$ for 7 and 26 Tesla respectively.

The parameters from x-ray and Raman data analysis are collected in Table I. The alignment fractions from 2-D and 3-D models differ by only a few percent. This implies that the Raman intensity is much less sensitive to out-of-plane than in-plane misalignment when the polarized measurement is done in-plane. The sensitivity analysis in Table I shows that even the 2-D model gives a reasonable estimate for $A_{R}$. Note that the $A_{R}$ 's are much larger than $\mathrm{x}$-ray-derived $\mathrm{A}_{\mathrm{X}}$, the latter being corrupted by unoriented non-SWNT impurities as noted above. Also note that for a given model, the aligned fractions are systematically 7$10 \%$ greater at $26 \mathrm{~T}$ compared to $7 \mathrm{~T}$. Absolute values are subject to uncertainties of order \pm 0.05 due to large experimental error bars and the lack of an exact value for $\mathrm{A}_{\mathrm{R}}{ }^{*}$; our best estimates are $\sim 0.73$ and $\sim 0.79$ for 7 and 26 Tesla respectively. 


\section{TEXTURE SUMMARY AND DISCUSSION}

$\mathrm{X}$-ray scattering gives a good estimate of the in-plane distribution width, while Raman scattering specifies the aligned fraction within a narrow range. Combining the two, we can compare texture parameters for $7 \mathrm{~T}$ and $26 \mathrm{~T}$ aligned buckypapers, Table I. The first striking feature is that there is no significant difference between the distribution widths at 7 and 26 Tesla. This suggests that the quality of SWNT alignment in the deposited film after removal from the filter membrane is limited by something other than the strength of the magnetic field. If alignment is limited by the degree of dispersion and disentanglement of the raw material in suspension, then 7 Tesla would provide sufficient torque to align the large ropes which are most likely to break free from the tangles. Another possible limiting factor could be turbulence at the interface between the suspension and the growing film, in which case further reduction in FWHM requires improvement in the hydraulics of filter deposition. It is interesting to note that the

FWHM of a $1 \mu \mathrm{m}$ thick 26 Tesla sample is only $25^{\circ}$ [6], suggesting that the initial deposit is better aligned than subsequently grown material.

The FWHM's are not significantly different for the two fields, while the aligned fraction is slightly larger at 26 Tesla. This is consistent with a crossover field somewhere between 7 and 26 Tesla such that more single tubes and other small objects become aligned at the higher field. 


\section{ELECTRICAL PROPERTIES}

In this section we address the consequences of magnetic field alignment on the electrical resistivity $\rho$, which we expect to exhibit anisotropy reflecting the SWNT alignment. We further expect the magnitude of the parallel component to be significantly smaller than the directionally-averaged $\langle\rho\rangle$ of random mats or buckypaper made from similar SWNT material.

A very crude estimate of $\rho / /$ provides a target for comparison with experimental results. Assume that each metallic tube in a perfectly aligned sample is comprised of finite length 2-channel ballistic conductors in series, the length being the mean free path for electron-phonon backscattering at some temperature. Taking $300 \mathrm{~nm}$ for this length at $300 \mathrm{~K}[18], \quad \rho_{/ /} \sim($ area/tube $) /\left(2 \mathrm{G}_{\mathrm{O}} \times 300 \mathrm{~nm}\right) \sim 1.5 \times 10^{-5} \Omega-\mathrm{cm}$ where $\mathrm{G}_{\mathrm{O}}$ is the

conductance quantum $(12.6 \mathrm{k} \Omega)^{-1}$. Assuming further that tube growth is stochastic with respect to wrapping indices, only $1 / 3$ of the tubes will be metallic at $300 \mathrm{~K}$ so $\rho / /$ $\sim 5 \times 10^{-5} \Omega$-cm., roughly twice the value for graphite. Factors which will increase this number in real samples include finite distribution width of tube axes, unaligned tubes, empty volume (porosity), junction resistance between tube segments and between ropes, incoherent inter-tube scattering within a rope and elastic scattering from tube ends, defects and impurities. These may be partially offset by p-doping of the semiconducting tubes by acid residues from purification and by atmospheric oxygen. Approximate corrections for porosity are straightforward [19]. 
In Figure 6 we plot 4-probe dc measurements of $\rho(T)$ for separate samples of annealed 7 Tesla material with in-line contacts parallel and perpendicular to $\mathrm{H}$. Resistivity values are based on actual sample dimensions and are subject to $15 \%$ uncertainties of the film thickness. The temperature dependence is weakly non-metallic and quite similar for both orientations. The anisotropy ratio $\rho_{\perp} / \rho_{/ /}$decreases gradually from $\sim 9$ to $\sim 8$ as $\mathrm{T}$ increases from 1.3 to $295 \mathrm{~K}$. The nonmetallic T-dependence is consistent with previous results on unoriented buckypaper after similar acid purification and high temperature vacuum annealing [12]. At $295 \mathrm{~K}$ the measured $\rho / /$ is $0.91 \mathrm{~m} \Omega-\mathrm{cm}$ based on measured dimensions. The measured density is $0.6 \mathrm{~g} / \mathrm{cm}^{3}$, less than half the value for a crystal of $1.3 \mathrm{~nm}$ diameter tubes, so the density-corrected resistivity is $0.41 \mathrm{~m} \Omega-\mathrm{cm}$. This accounts approximately for gross porosity.

Similar data for the 26 Tesla-aligned material is shown in Figure 7, where we compare results before and after annealing. For the as-deposited sample $\mathrm{d} \rho / \mathrm{dT}$ is positive above $\sim 200 \mathrm{~K}$ whereas after annealing it remains negative up to $295 \mathrm{~K}$, as for the annealed 7 Tesla sample. Absolute values for the as-deposited sample are 10 times smaller for both orientations compared to the annealed sample. Both observations can be explained by charge transfer doping from acid purification residues which are eliminated during annealing (26\% weight loss) [12]. The anisotropy is $\sim 6$, somewhat less than at 7 Tesla, independent of temperature and annealing. The density after annealing is 
$\sim 0.9 \mathrm{~g} / \mathrm{cm}^{3}$ so the volume-corrected $\rho / /$ after annealing is $0.55 \mathrm{~m} \Omega-\mathrm{cm}$.

Table II summarizes the density-corrected resistivity at $295 \mathrm{~K}$ The apparently smaller anisotropy ratio at 26 compared to 7 Tesla is in fact within error bars. The large differences between as-grown and annealed resistivities are attributed to p-doping during acid purification. No attempt was made to exclude oxygen because this gives only a $25 \%$ effect on $\rho$ [20]. Annealing does not affect the resistivity anisotropy, while x-ray diffraction reveals a large improvement in crystallinity [6].

If the alignment were perfect, we would expect different transport mechanisms for the two orientations with different temperature dependences. Since the alignment is not perfect, current flow is primarily along tubes and/or ropes for both macroscopic orientations. The intrinsic anisotropy is no doubt much greater than what is exhibited by this sample. In particular since we expect $\rho_{/ /}<\rho_{\perp}$, the unaligned fraction provides low-resistance shorts with $\mathrm{E} \perp \mathrm{H}$. To quantify the effect of alignment on resistivity anisotropy, we model the oriented buckypaper as an ensemble of 1-D paths in the plane of the sample, each containing on average $n$ elements (ropes, tubes) of fixed length and resistance. The resistance of each path is linearly proportional to $n$, and since for a fixed number of elements the number of paths is inversely proportional to $n$, the resistance of the ensemble of paths in parallel is proportional to $n^{2}$. The average number of elements $n$ required for current to flow through the sample is equal to $L \wedge$, where $L$ is the length of the sample and $\lambda$ is the mean projection of the element length onto the current direction (assuming the orientation distribution function $\mathrm{F}(\Psi, \Delta \Psi)$ is symmetric with respect to this 
direction, as is certainly true for measurements of $\rho / /$ and $\rho \perp$ ). Thus the average number of elements in the path for current flow parallel to $\mathrm{H}$ is $n_{\|} \propto \frac{1}{\langle\cos (\Psi)\rangle}$, which incorporates the experimental distribution function via

$$
\langle\cos (\Psi)\rangle=\int_{\frac{-\pi}{2}}^{\frac{\pi}{2}} \cos (\Psi) F(\Psi, \Delta \Psi) d \Psi
$$

and similarly for $n_{\perp} \propto \frac{1}{\langle|\sin (\Psi)|\rangle}$. We estimate the anisotropy in resistivity due to alignment, $\frac{\rho_{\perp}}{\rho_{\|}}=\left|\frac{\langle\cos (\Psi)\rangle}{\langle|\sin (\Psi)|\rangle}\right|^{2}$, using either x-ray or Raman texture data from Table I to specify F. The results are included in Table II. Using the Raman data for 26 Tesla, the agreement is almost perfect. On the other hand, $\mathrm{x}$-ray data input yields resistivity ratios which are $\sim 1 / 3$ and $1 / 2$ the experimental values at 7 and 26 Tesla respectively, due no doubt to the poor accuracy of $A_{X}$ compared to $A_{R}$. At 7 Tesla the experimental ratio is $30 \%$ greater than the one calculated using Raman texture data, which could be attributed to an experimental overestimate of $\rho \perp$ due to incipient cracks in the sample. In general, polarized Raman measurements give much better estimates of resistivity ratios than x-ray data because $A_{R}$ is more reliable than $A_{X}$.

The same approach can be used in reverse to estimate the x-ray aligned fraction $A_{X}$ from the experimental resistivity ratio and the quite reliable $x$-ray FWHM. The results are 0.73 and 0.65 at 7 and 26 Tesla respectively. These are in much better 
agreement with the experimental $A_{R}$ 's than with the experimental $A_{X}$ 's, indicating once again that the $\mathrm{x}$-ray diffuse scattering contains a large contribution from non-SWNT scatterers.

After annealing out the acid dopants, the lowest density-corrected $\rho / /, 4.1 \times 10^{-4}$ $\Omega$-cm, is within a factor $\sim 10$ of our $5 \times 10^{-5} \Omega$-cm benchmark. Improvements in mosaic width and aligned fraction should lead to reduced values of $\rho_{/ /}$. The rather surprising inference is that interparticle contacts do not play a dominant role in limiting the $300 \mathrm{~K}$ resistivity of bulk SWNT materials. A direct comparison between $\rho / /$ and $<\rho>$ from an isotropic sample cannot be made since we do not have an adequate unaligned control sample.

\section{THERMAL CONDUCTIVITY}

Figure 8 shows $\kappa(T)$ for 7 and 26 Tesla measured with the heat flow Q parallel and perpendicular to the magnetic field axis on separate samples. The room temperature density-corrected values are included in Table II. Measurements from 10 to $300 \mathrm{~K}$ were performed using a comparative technique [21]. A known heat flow Q passed through a constantan rod, then the sample, and finally a second constantan rod, to a heat sink. The sample conductance was measured by averaging the two ratios of temperature drops along either constantan and sample, and then scaling by a dimensional factor; finally $\kappa$ (sample) was obtained by scaling to the known value for constantan. Three differential Type E thermocouples using 0.00025 inch diameter wires were employed to measure 
temperature drops across the sample and the constantan standards. Small sapphire chips were used to electrically isolate the differential thermocouple from the sample. The averaging of two ratios accounts approximately for radiation losses, since the one of the two constantans is hotter or colder than the sample.

The thermal conductivity increases smoothly with increasing temperature, and displays a temperature dependence similar to that of unoriented SWNT [7,21]. The monotonic increase with increasing $\mathrm{T}$ reflects the thermal population of phonon states, this being the dominant heat conducting mode even in metallic tubes. The values of $\kappa / /(300 \mathrm{~K})$ are similar for both 7 and 26 Tesla samples, while one might expect a higher value at 26 Tesla if the alignment were better [22]. In fact the $\kappa$ results are consistent with the texture analysis which shows that the two samples do not differ significantly with respect to the degree of alignment. Note also that the anisotropy ratios for $\kappa(300 \mathrm{~K})$ in Table II are essentially the same as the resistivity anisotropies. This suggests that the "shorting" effect of misaligned tubes and ropes is similar for electron and phonon transport.

\section{SUMMARY}

The anisotropic morphology of magnetic field-aligned SWNTs leads to anisotropic electrical and thermal transport properties. Alignment of the SWNTs increases the parallel components of both the electrical and thermal conductivity with respect to unoriented material. In the parallel direction, the room-temperature electrical 
conductivity is comparable to individual SWNT ropes. Further enhancements in transport anisotropies should focus on optimization of the alignment/filter deposition process since there is no significant advantage of 26 Tesla compared to 7 Tesla. We show that both Raman and x-ray data are best described by including a fraction of unaligned material, and that the best way to determine both the distribution width and aligned fraction is to combine x-ray and Raman data in order to decouple the respective fitting parameters.

\section{ACKNOWLEDGMENTS}

This work was supported by the National Science Foundation MRSEC Grant DMR00-79909 (WZ, Raman and x-ray scattering), Office of Naval Research Grant N00014-00-1-0720 (RH, x-ray scattering), the US Department of Energy Grant DEFG02-98ER45701 (JV and MCL, transport properties), award No. NCC 9-77 from the National Aeronautics and Space Administration, ONR grant N00014-99-1-0246 and the Welch Foundation (MJC, synthesis). 


\section{REFERENCES}

1. L. Jin. C. Bower and O. Zhou, Appl. Phys. Lett. 3, 1197 (1998).

2. R. Haggenmueller, H. H. Gommans, A. G. Rinzler, J. E. Fischer and K. I. Winey, Chem. Phys. Lett. 330, 219 (2000).

3. B. Vigolo, A Penicaud, C. Coulon, C. Sauder, R. Pallier, C. Journet, P. Bernier and P. Poulin, Science 290, 1331 (2000).

4.. P. Launois, A. Marucci, B. Vigolo, A. Derre and P. Poulin, J. Nanoscience and Nanotechnology Vol. 1 (2001) pp125-128.

5. D. A. Walters, M. J. Casavant, X. C. Qin, C. B. Huffman, P. J. Boul, L. M. Ericson, E. H. Haroz, M. J. O'Connell, K. Smith, D. T. Colbert, and R. E. Smalley, Chem. Phys. Lett. 338 (2001) $14-20$.

6. B. W. Smith, Z. Benes, D. E. Luzzi, J. E. Fischer, D. E. Walters, M. J. Casavant, J. Schmidt and R. E. Smalley, Appl. Phys. Lett. 77, 663 (2000).

7. J. C. Hone, M. C. Llaguno, N. M. Nemes, J. E. Fischer, D. E. Walters, M. J. Casavant, J. Schmidt and R. E. Smalley, Appl. Phys. Lett. 77, 666 (2000).

8. M. J. Casavant, D. A. Walters, J. J. Schmidt and R. E. Smalley, to be published.

9. H. H. Gommans, J. W. Alldredge, H. Tashiro, J. Park, J. Magnuson and A. G. Rinzler, J. Appl. Phys. 88, (2000) 2509.

10. N. Coustel, N. Foxonet, J. L. Ribet, P. Bernier and J. E. Fischer, Macromolecules 24, 5867 (1991).

11. J. E. Fischer, Q. Zhu, X. Tang, E. M. Scherr, A. G. MacDiarmid and V. B. Cajipe, Macromolecules 27, 5094 (1994). 
12. A. G. Rinzler, J. Liu, P. Nikolaev, C. B. Huffman, F. J. Rodriguez-Macias, P. J. Boul, A. H. Lu, D. Heymann, D. T. Colbert, R. S. Lee, J. E. Fischer, A. M. Rao, P. C. Eklund, and R. E. Smalley, Applied Physics A 67, 29 (1998).

13. A. Thess, R. Lee, P. Nikolaev, H. Dai, P. Petit, J. Robert, C. Xu, H. Lee, S.G. Kim, D. T. Colbert, G. Scuseria, D. Tomanek, J. E. Fischer and R. E, Smalley, Science 273， 483 (1996).

14. S. Rols, R. Almairac, E. Anglaret and J. L. Sauvajol, Euro. Phys. J. B 10, 263 (1999).

15. http://www.Irsm.upenn.edu/lrsm/facMAXS.html

16. E. Anglaret,, A. Righi, J. L. Sauvajol, P. Bernier, B. Vigolo and P. Poulin, Phys. Rev. B 65, 165426 (2002).

17. The value of $56^{\mathrm{o}}$ mentioned in Ref. 6 was not corrected for absorption or scattering volume and is therefore only a gross upper limit.

18. M. Radosavljevic, $\mathrm{PhD}$ thesis, University of Pennsylvania (2001).

19. J. E. Fischer, H. Dai, A. Thess, R. Lee, N. M. Hanjani, D. DeHaas and R. E. Smalley, Phys. Rev. B 55, R4921 (1997).

20. G. U. Sumanasekera, C. K. W. Adu, S. Fang and P. C. Eklund, Phys. Rev. Letters 85, 1096 (2000).

21. M. C. Llaguno, J. Hone, A.T. Johnson and J. E. Fischer, in Molecular Nanostructures, edited by H. Kuzmany, J. Fink, M. Mehring and S. Roth, AIP Conf. 22. In Ref. 7 we reported a $300 \mathrm{~K}$ density-corrected $\kappa / /$ value of $200 \mathrm{~W} / \mathrm{mK}$, 4 times greater than given in Table II. In the earlier work, the absolute value was obtained by scaling the electrical resistance of the $\kappa$ sample to that of a similar sample with betterdefined geometry, assuming the resistivities were the same. The discrepancy between the 
two scaling methods could be attributed to differences in resistivity for nominally identical samples. 
Table I. Summary of texture analysis fit parameters for magnetically-aligned buckypapers using 7 and $26 \mathrm{~T}$ fields. FWHM and $\mathrm{A}_{X}$ are the widths of Gaussian distributions of SWNT axes with respect to the aligning magnetic field axis and aligned fractions determined from $x$-ray scattering. $A_{R}$ are the aligned fractions deduced from Raman fits to various models as noted.

\begin{tabular}{|c|c|c|c|c|}
\hline & $\begin{array}{c}\text { XRAY } \\
\text { FWHM } \\
\text { (deg.) }\end{array}$ & $A_{X}$ & RAMAN MODEL & $A_{R}$ \\
\hline \multirow{5}{*}{7 Tesla } & \multirow{5}{*}{$33.0 \pm 0.2$} & \multirow{5}{*}{0.42} & $2-\mathrm{D}$ & 0.71 \\
\hline & & & 3-D, out-of plane $\mathrm{A}_{\mathrm{R}}{ }^{*}=1, \mathrm{FWHM}^{*}=10$ & $\overline{0.71}$ \\
\hline & & & 3 -D, out-of plane $\mathrm{A}_{\mathrm{R}}^{*}=1, \mathrm{FWHM}^{*}=30$ & 0.72 \\
\hline & & & 3 -D, out-of plane $\mathrm{A}_{\mathrm{R}}^{*}=0.8, \mathrm{FWHM}^{*}=10$ & $\overline{0.73}$ \\
\hline & & & 3 -D, out-of plane $\mathrm{A}_{\mathrm{R}}^{*}=0.8, \mathrm{FWHM}^{*}=30$ & 0.74 \\
\hline \multirow{5}{*}{26 Tesla } & \multirow{5}{*}{$34.0 \pm 0.5$} & \multirow{5}{*}{0.40} & $2-\mathrm{D}$ & $\overline{0.78}$ \\
\hline & & & 3 -D, out-of plane $\mathrm{A}_{\mathrm{R}}^{*}=1, \mathrm{FWHM}{ }^{*}=10$ & 0.78 \\
\hline & & & 3-D, out-of plane $A_{R}^{*}=1, F_{H H M}^{*}=30$ & 0.79 \\
\hline & & & 3-D, out-of plane $\mathrm{A}_{\mathrm{R}}^{*}=0.8, \mathrm{FWHM}^{*}=10$ & 0.79 \\
\hline & & & 3-D, out-of plane $\mathrm{A}_{\mathrm{R}}{ }^{*}=0.8, \mathrm{FWHM}^{*}=30$ & $\overline{0.80}$ \\
\hline
\end{tabular}


Table II. . Summary of $295 \mathrm{~K}$ resistivity $\rho$ and thermal conductivity $\kappa$ measurements on oriented SWNT films. Values are corrected approximately for gross porosity by scaling the raw data by the fraction of ideal density. Anisotropy ratios $\rho_{\perp} / \rho_{/ /}$and $\kappa_{/ /} / \kappa_{\perp}$ are also given, as well as $\rho_{\perp} / \rho_{/ /}$ratios predicted by a simple network model using $\mathrm{X}$-ray or Raman texture parameters as inputs.

\begin{tabular}{|c|c|c|c|}
\hline & $\begin{array}{c}7 \text { TESLA } \\
\text { ANNEALED }\end{array}$ & $\begin{array}{c}26 \text { TESLA } \\
\text { AS-GROWN }\end{array}$ & $\begin{array}{c}26 \text { TESLA } \\
\text { ANNEALED }\end{array}$ \\
\hline$\rho_{\perp}$ & $3.3 \mathrm{~m} \Omega-\mathrm{cm}$ & $0.52 \mathrm{~m} \Omega-\mathrm{cm}$ & $3.4 \mathrm{~m} \Omega-\mathrm{cm}$ \\
\hline$\rho_{/ /}$ & $0.41 \mathrm{~m} \Omega-\mathrm{cm}$ & $0.085 \mathrm{~m} \Omega-\mathrm{cm}$ & $0.55 \mathrm{~m} \Omega-\mathrm{cm}$ \\
\hline $\begin{array}{c}\rho_{\perp} / \rho_{/ /} \\
\text {EXPERIMENTAL }\end{array}$ & 8.0 & 6.1 & 6.2 \\
\hline $\begin{array}{c}\rho_{\perp} / \rho_{/ /} \\
\text {PREDICTED FROM } \\
\text { X-RAY } \\
\end{array}$ & 3.0 & & 2.8 \\
\hline $\begin{array}{c}\rho_{\perp} / \rho_{/ /} \\
\text {PREDICTED FROM } \\
\text { RAMAN } \\
\end{array}$ & 6.0 & & 6.2 \\
\hline$\kappa_{\perp}$ & $5 \mathrm{~W} / \mathrm{m} \mathrm{K}$ & & $8 \mathrm{~W} / \mathrm{m} \mathrm{K}$ \\
\hline$\kappa / /$ & $48 \mathrm{~W} / \mathrm{m} \mathrm{K}$ & & $42 \mathrm{~W} / \mathrm{m} \mathrm{K}$ \\
\hline$\kappa_{/ /} / \kappa_{\perp}$ & 10 & & 5 \\
\hline
\end{tabular}




\section{FIGURE CAPTIONS}

Fig. 1. Wide-angle x-ray diffraction from 7 Tesla-aligned SWNT buckypaper (raw data, no background subtraction). Sample is a film in reflection geometry. Beam divergence

normal to the diffracting plane is estimated at $\pm 2^{\mathrm{O}}$. Vertical divergence integrates the scattered beam over $\sim \pm 2^{\circ}$ in $\chi$.

Fig. 2. Background-subtracted X-ray counts, summed over intervals $5.1^{\mathrm{o}}<2 \theta<7.5^{\mathrm{o}}$ about the $(1,0)$ rope reflection, every $1^{0}$ in $\chi$. Data are the symbols; fits to two Gaussians plus a constant are the smooth curves. Magnetic fields 7 Tesla (a) and 26 Tesla (b). Intensity scales are arbitrary.

Fig. 3. Similar to Figure 2 but with the $2 \theta$ window shifted below the Bragg peak to isolate texture in the diffuse scattering.

Fig. 4. Polarized Raman spectra of 7 Tesla film measured with $\Psi$ (angle between polarization vector and $\mathrm{H}$ direction) at $0,45^{\circ}$ and $90^{\circ}$. Note that all Raman features vary similarly with $\Psi$.

Fig. 5. Analysis of polarized Raman spectra. Symbols are Raman $\mathrm{G}_{2}$-band intensities, averaged over 5 spots on the sample, versus $\Psi$ for aligned nanotubes at 7 and 26 Tesla, (a) and (b) respectively. Solid curves are best fits from the anisotropic 3-D model, obtained by optimizing the aligned fraction and fixing the distribution width at the $\mathrm{x}$-ray value from Table I.

Fig. 6. Resistivity vs. temperature for annealed 7 Tesla-aligned buckypaper, measured with current perpendicular and parallel to the average alignment axis on two different samples with in-line 4-probe contacts.. 
Fig. 7. As in Figure 6 for 26 Tesla alignment, including measurements before (a) and after annealing (b).

Fig. 8. Thermal conductivity of annealed 7 (a) and 26 Tesla-aligned SWNT films (b) measured with heat flow perpendicular and parallel to the alignment axis. 